\title{
1 Three-dimensionality of shallow island wakes
}

\author{
2 Paul M. Branson - Marco Ghisalberti . \\ 3 Gregory N. Ivey
}

4

Received: DD Month YEAR / Accepted: DD Month YEAR

\begin{abstract}
Island wakes are thought to play a significant role in the vertical and cross shelf mixing processes in strong tidally forced coastal regions. This paper describes a comprehensive laboratory study of shallow water wakes behind islands of circular cross section forced by a sinusoidal tidal flow. The wake structure and vertical circulation are determined through novel three-dimensional particle imaging velocimetry measurements. Four archetypal wake forms (symmetric, asymmetric, unsteady bubble, and vortex shedding) are observed. Through examination of the vertical structure of each of these wake forms, we demonstrate the dependence of vertical transport in island wakes on three key parameters: i) the tidal excursion relative to the island size, ii) the bottom boundary layer thickness relative to the flow depth and iii) the aspect ratio of the island size to the flow depth. The importance of secondary vortices in island upwelling is highlighted by local peaks in vertical velocity that exceed $40 \%$ of the peak external tidal velocity. This study fundamentally changes the view of island wake upwelling from a weak 'tea cup'-like recirculation process to one where primary and secondary flow structures vigorously stir the water column over the full depth. This has fundamental implications for the fate of passive biological tracers and the time scales that determine productivity in topographically-complex continental shelf regions.
\end{abstract}

P. M. Branson

Ocean Graduate School

University of Western Australia

35 Stirling Hwy

Crawley, Australia, 6009

E-mail: paul.branson@research.uwa.edu.au

M. Ghisalberti

Department of Infrastructure Engineering

University of Melbourne

Parkville, Australia, 3010

G. N. Ivey

Ocean Graduate School

University of Western Australia

35 Stirling Hwy

Crawley, Australia, 6009 
Keywords Island Wakes · Three Dimensional Flow · Tidal Forcing · Upwelling · Vorticity

\section{Introduction}

The wakes of islands and headlands play an important role in lateral and vertical mixing processes, with implications for the transport of nutrients, plankton, sediment and pollutants. The vertical transport (upwelling) of nutrient rich water into the surface layer (that has higher light intensity) has important ecological implications due to the resulting increased primary productivity. Numerous studies have identified the importance of complex flow features such as eddies and fronts (shear zones) for aggregating sediment and plankton [35, 15, 23]. This can, in turn, effect the distribution of benthic and higher order pelagic organisms that graze along these fronts and wakes, taking advantage of lower-trophic-level aggregations $[34,16,17]$. Despite the perceived ecological importance of island wakes, they have received only intermittent research attention over the past several decades, and to date no clear mechanistic description of the flow has clearly supported the hypothesised ecological importance.

\subsection{Variation of island wake form}

One of the most interesting features of island wakes is the insensitivity of the large scale flow features to the Reynolds number, $\operatorname{Re}=U D / \nu$, where $U$ is the characteristic flow speed, $D$ the characteristic island diameter and $\nu$ the molecular kinematic viscosity (or eddy viscosity for turbulent flow [10]). For flow around a cylinder in the laboratory a Kármán vortex street is observed for Re $\gtrsim 40$, which is beautifully pictured on the cover of the Album of Fluid Motion [30]. The Kármán vortex street observed in the laboratory island wake is an analogue of vortex streets observed in atmospheric and oceanic island wake flows, as seen in cloud formations downwind of the Canary Islands and Madeira near north Africa (Fig. 1). Various studies have noted the structural similarity of field scale wakes to laboratory island wakes at a significantly lower Reynolds number. In the oceanic wake of Rattray Island in the Australian Great Barrier Reef the turbulent horizontal eddy viscosity was estimated from the dispersion of floating drogues as $O(1) \mathrm{m}^{2} \mathrm{~s}^{-1}$ with a peak Reynolds number of order $10^{3}$ [35]. If the flow were unbounded this implies a vortex shedding wake, however the attached recirculating wake is structurally more similar to a wake of $\mathrm{Re} \lesssim 20$ [37]. As the flow depth is reduced, the vorticity generated in the bottom boundary layer increasingly distorts the vertical vorticity generated in the lateral shear layer to the point where the flow becomes completely dominated by bottom friction in shallow flow. Therefore in shallow island wake flow, the Reynolds number is not expected to be the controlling parameter.

At the flanks of the island, transverse shear is present due to the island boundary. Linear stability analyses of laminar parallel shear flows in shallow water led to the introduction of a bottom friction stability parameter that represents the ratio of the stabilizing effect of bed friction and the destabilizing effect of transverse shear [11]. Efforts to predict the onset of vortex shedding in island wakes and the qualitatively observed wake forms lead to the extension of the bottom 


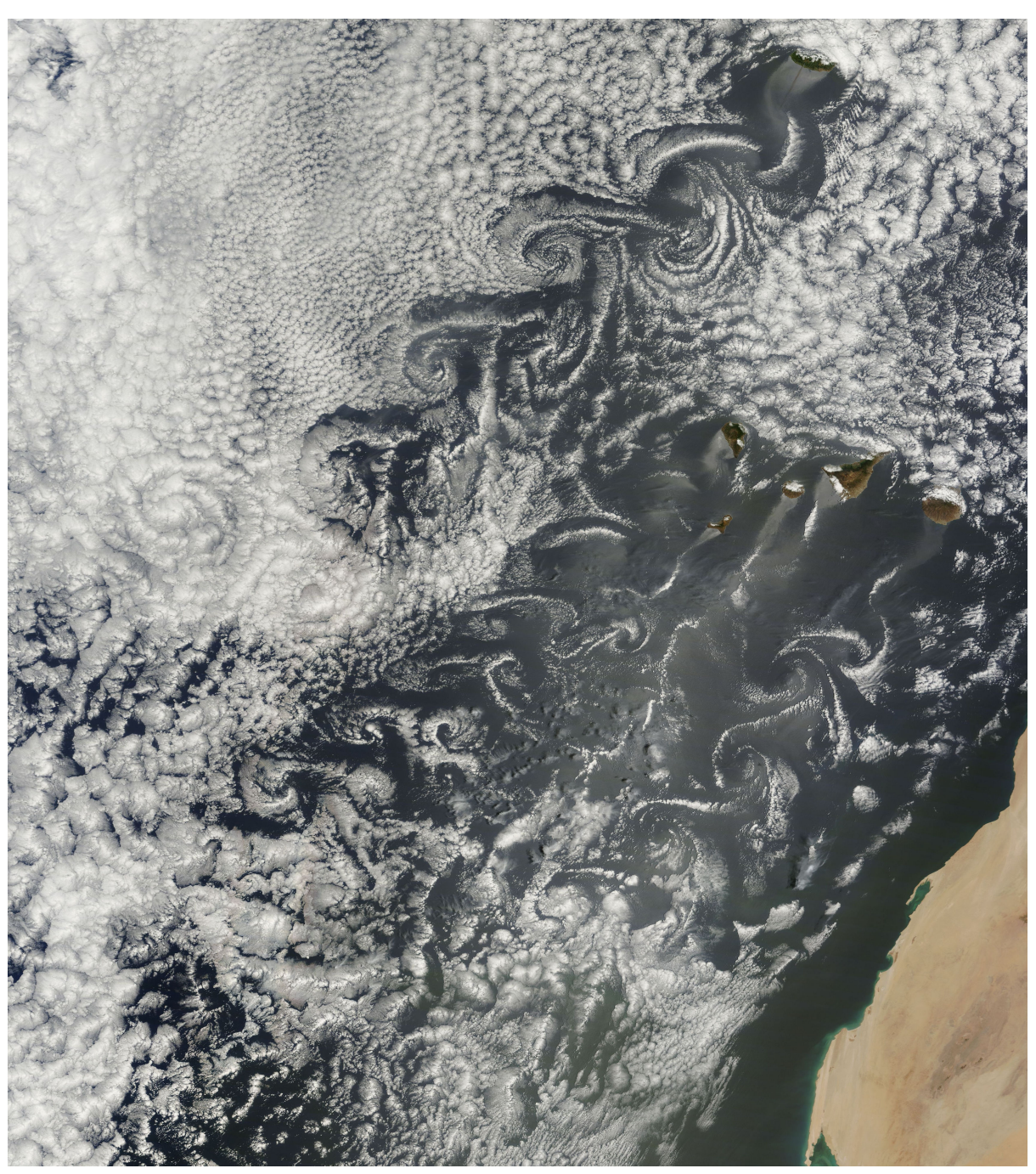

Fig. 1 MODIS/Terra image of multiple Kármán vortex streets swirling downwind of the Canary Islands and Madeira, May 20, 2015. (Image courtesy of NASA/Goddard Space Flight Center Earth Science Data and Information System (ESDIS) project.)

friction stability parameter to bluff body wake flow resulting in the wake stability parameter $S=c_{f} D / h$ where $c_{f}$ is a friction coefficient and $h$ is the water depth [15] (or, alternatively, the island wake parameter $P_{I} \sim 1 / S$ [35]). An extensive laboratory study of shallow turbulent wakes in steady flow identified three classes of wake form: (i) the vortex street, (ii) the unsteady bubble wake and (iii) the steady bubble wake, with the transitions between the different wake forms well predicted by $S[9]$.

Despite the success of $S$ in predicting the wake form at the laboratory scale, applications at the field scale have had limited success in predicting island wake forms in oceanic flows $[15,14]$. This discrepancy has typically been attributed to 
the unsteadiness of tidal currents, which causes strong temporal fluctuation in the wake structure. In contrast to atmospheric island wake flows (e.g. Fig. 1), oceanic island wakes on continental shelves are indeed subjected to unsteady tidal forcing. In steady shallow flows, bottom friction is felt over the entire depth. In oscillating flows, however, the Stokes boundary layer thickness can be small compared to the total depth, thus restricting frictional effects to only a portion of the water depth.

In addition to the influence of flow unsteadiness in altering the vertical profile of horizontal velocity, flow reversal means that the wake generated earlier on one side of the island can interact with the newly-forming wake on the other side. The effects of this half-cycle interaction have been extensively studied in unbounded sinusoidal flow around circular cylinders [33, 25]. In its simplest form, tidal flow can modelled as a sinusoidally-varying flow of amplitude $U_{0}$ and period $T$. The Keulegan-Carpenter number, $K C=U_{0} T / D$, predicts the onset of vortex shedding and an extensive variety of wake forms have been observed to be dependant on the relative value of the time scale of the sinusoidal flow $(T)$ and the time scale of wake vortex evolution $\left(D / U_{0}\right)$. The different wake forms observed in unbounded sinusoidal flow around a cylinder are due to a range of complex vortex pairing patterns where the vortex from one half-cycle is advected back past the cylinder to pair with a newly generated vortex on the next half-cycle.

The influence of flow unsteadiness on shallow island wakes has been investigated in laboratory experiments that examined the combined effects of $K C$ and $S$ on wake form and evolution [21]. The observed wake forms were classified (in order of increasing wake complexity) as: symmetric-without-pairing, symmetricwith-pairing, sinuous-with-pairing and vortex-shedding [21]. Whilst general trends in wake 'stability' were observed with increasing $S$ and decreasing $K C$, these parameters alone did not clearly delineate the observed wake forms. A $2 \mathrm{D}$ numerical study of shallow headland wakes observed that wake behaviour was best predicted by the ratio of two length scales, the tidal excursion length $l_{t}=U_{0} T / 2 \pi$ and the frictional length $l_{f}=h / c_{f}$ [29]. When $l_{f}>l_{t}$, stable wake forms were observed; conversely, when $l_{t}>l_{f}$, stability in the wake was reduced and vortex shedding was often observed. For shallow, laminar, oscillatory flow around islands, $K C$ and $S$ are inter-related according to

$$
S \sim \frac{\delta^{+}}{K C}
$$

where $\delta^{+}=\sqrt{\nu T} / h$, with $\sqrt{\nu T}$ the characteristic length scale associated with the Stokes boundary layer $[7,8]$. Furthermore, $\delta^{+}$is equivalent to $l_{t} / l_{f}$, the ratio observed to predominantly govern headland wake behaviour in tidal flow [29]. The parameter $\delta^{+}$, a non-dimensional Stokes boundary layer thickness, quantifies the relative influence of momentum diffusion to the bed in a shallow oscillatory flow. The two parameters $K C$ and $\delta^{+}$that determine the stability parameter $S$ in oscillatory island wake flow, can be independently varied and allows us to examine the influence of relative wake length and bed friction on the wake of a shallow island in a tidal flow. 
1.2 Circulation of island wake eddies

Whilst prediction of the horizontal structure is an important aspect of the study of island wakes, the fundamentally-important process from an ecological perspective is the vertical transport within the wake. Few studies have obtained direct measurements of vertical velocity in island wakes, due primarily to the non-stationary and three-dimensional character of these flows. Laboratory measurements of steady attached island eddies suggested that downwelling occurred on the eddy periphery, with upwelling in the eddy core (with a magnitude of up to $1.5 \%$ of the tidal velocity) [36]. This evoked the steady 'tea-cup' analogy [36] often applied in geophysical fluid dynamics. The 'tea-cup' analogy is where rotation in the island eddy establishes a radial pressure gradient at the free surface (due to centrifugal acceleration). The free surface pressure is transmitted through the bottom boundary layer, and drives a convergent boundary layer flow that upwells in the eddy core. Whilst this interpretation was supported by dye observations of upwelling in the core of the primary Kármán vortices, detailed planar PIV measurements demonstrated that the vertical motion rolls up into a secondary horizontal vortex [1]. Shadowgraph visualisation demonstrated that these secondary vortices can induce significant free surface perturbations, despite their circulation being an order of magnitude smaller than the primary vertical vortices [1]. Although this demonstrated a fundamentally three-dimensional flow structure, the implications of the secondary vortices for upwelling in island wake flows was not recognised.

Further insight into the three-dimensionality of the wake can be obtained through the study of shallow vortex dynamics. In addition to the external scales governing the overall wake stability (i.e. tidal excursion, island size, flow depth and tidal boundary layer thickness), there are inherent wake vortex scales that govern the evolution of three-dimensionality. Planar stereoscopic PIV measurements through a supposedly quasi-two-dimensional, shallow di-polar vortex revealed the evolution of a horizontal secondary vortex with significant associated vertical velocity [2]. The vertical recirculation was an intrinsic feature of the vertically-confined flow, rather than the result of the no-slip boundary at the bed [2]. An analytical and numerical study of shallow swirling vortex flows demonstrated that the threedimensionality of, and upwelling regime within, a mono-polar vortex is controlled by a relative boundary layer thickness [12]:

$$
h_{R e}=\frac{2}{\left(h / D_{v}\right) \operatorname{Re}^{1 / 2}} \equiv 2 \sqrt{\nu D_{v} / U_{v}} / h
$$

where $U_{v}$ and $D_{v}$ are the characteristic azimuthal velocity and diameter (respectively) of the mono-polar vortex. As the ratio $D_{v} / U_{v}$ represents the time scale for vortex rotation, it is clear that $\delta^{+}$and $h_{R e}$ are conceptually equivalent parameters that describe the relative influence of vertical diffusion of momentum to the bed. In the viscous regime $\left(h_{R e} \gtrsim 0.6\right)$, vertical diffusion dominates, a Poiseuille-like vertical profile evolves in the azimuthal velocity, the flow is considered quasi-two-dimensional, and universal scaling relations for upwelling have been established [12]. In the inertial regime $\left(h_{R e} \lesssim 0.2\right)$, a distinct bottom boundary layer is present in the azimuthal velocity with an inviscid interior that allows the growth of non-linear inertial waves and the scaling of upwelling velocity depends on the initial vertical profile of velocity [12]. While there are clear similarities to 
isolated shallow vortices, island wake vortices are distinguished by their generation through entrainment across the free shear layers (due to flow separation), such that there is an interaction between the scales of the external tidal flow and those of the island wake vortices.

The objective of this study is to describe the vertical structure, three-dimensionality and upwelling in island wakes in a tidal flow. Through application of a novel threedimensional particle imaging velocimetry (3D PIV) approach we obtain (for the first time) a synoptic and time-resolved view of the three-dimensional wake circulation, and quantify the associated magnitude and spatial distribution of up- and downwelling in the near wake region. The governing parameters of $K C$ and $\delta^{+}$are varied independently to examine in detail four archetypal island wake forms.

\section{Experimental setup}

Experiments were undertaken in a shallow tidal flow flume specifically designed to produce an unsteady flow. An island of circular cross section and diameter $D=10 \mathrm{~cm}$ was utilised in this study. The flume had a working section $185 \mathrm{~cm}$ wide, $600 \mathrm{~cm}$ long and up to $35 \mathrm{~cm}$ deep (Fig. 2). The flume was recirculating and used a computer controlled variable frequency drive and pump to generate a reciprocating tidal flow with a near sinusoidal horizontal velocity signal. Polycarbonate flow straighteners were utilised in each end of the flume to produce a near uniform transverse velocity profile. The experimental flow conditions are detailed in table 1. Each flow was established for 10 cycles, before being sampled for 8 cycles.

Table 1 Conditions of the experimental runs, $K C=U_{0} T / D, \delta^{+}=\sqrt{\nu T} / h$ and $\operatorname{Re}_{D}=$ $U_{0} D / \nu$.

$\begin{array}{llllllll}\text { Wake type } & U_{0}\left[\mathrm{~cm} \mathrm{~s}^{-1}\right] & T[\mathrm{~s}] & h[\mathrm{~cm}] & \delta^{+} & K C & R e_{D} & h / D \\ \text { Symmetric } & 0.52 & 85.9 & 2.3 & 0.40 & 4.4 & 517 & 0.23 \\ \text { Asymmetric } & 0.39 & 121.6 & 4.3 & 0.26 & 4.8 & 394 & 0.43 \\ \text { Unsteady Bubble } & 2.76 & 85.9 & 2.3 & 0.40 & 23.7 & 2764 & 0.23 \\ \text { Vortex Shedding } & 2.12 & 121.6 & 4.3 & 0.26 & 25.8 & 2118 & 0.43\end{array}$

The flow was seeded with sieved Pliolite AC80 in the size range $300-450 \mu \mathrm{m}$ (an imaged particle size of approximately $3 \mathrm{px}$ ) and the working fluid (salt water) density matched to that of the seeding particles. Volumetric velocity measurements were obtained via synthetic aperture particle imaging velocimetry (PIV) [6]. An array of 9 Basler ACE acA1600-20gm 2.0 mega-pixel monochrome GigE cameras were positioned in a $3 \times 3$ grid arrangement approximately $110 \mathrm{~cm}$ below the flume with each camera aligned and focussed on a $34 \times 25 \times 6 \mathrm{~cm}^{3}$ measurement volume (figure 2). The camera array was calibrated using a multi-camera selfcalibration method that accounted for the air-glass-water refractive interfaces [5] with a resulting average reprojection error of $0.11 \mathrm{px}$. Due to the volume of the data generated by the camera array for the relatively long-running experiments (between 11 and 16 minutes), burst sampling was applied with 11 frames collected at $20 \mathrm{~Hz}$ each second. Camera synchronisation was achieved with a hardware timed trigger and the flow volume was illuminated using a high intensity array of LEDs. 


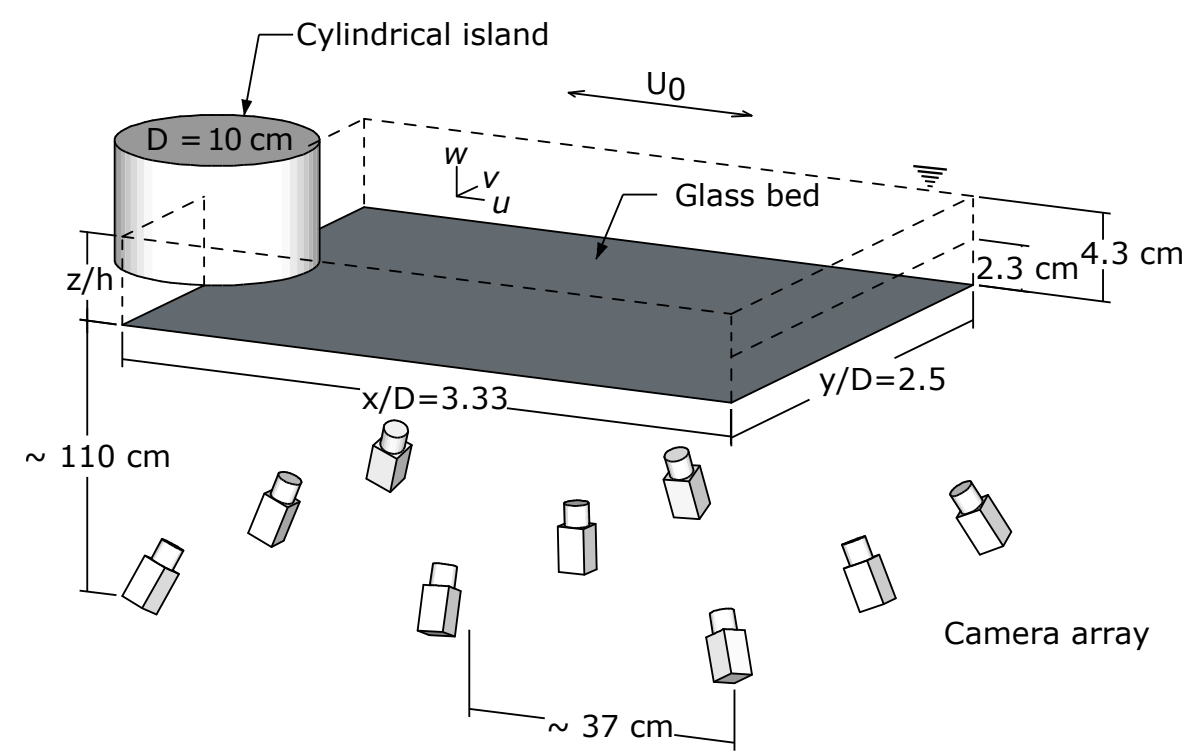

Fig. 2 Schematic diagram of the experimental setup with the measurement volume of the 3D PIV system indicated.

A 3D PIV analysis was performed using a multi-gridded, iterative, image deforming algorithm. The iterative analysis was applied for a total of 8 passes with the first three passes progressively refining the interrogation volume from $256 \mathrm{x}$ $256 \times 48$ voxels through to $96 \times 96 \times 12$ voxels with $75 \%$ overlap. The anisotropic interrogation volume was employed given that shallow flows are characterised by horizontal scales much larger than their vertical scales. Ensemble averaging was applied in the correlation space (across 5 image pairs) within each burst to reduce the influence of spatially-variable seeding density created by the converging and diverging flow. Sub-pixel displacement estimates were obtained using a three point Gaussian fit to the correlation peak in three dimensions. The universal outlier detection algorithm of [32] was applied after each pass to remove spurious vectors. Finally, a three-dimensional Gaussian filter $(\sigma=0.5)$ was applied to reduce noise. The inter-frame time for the analysis was adjusted for each experiment to minimise the outlier detection rate and the divergence error (discussed in the next section).

\subsection{Measurement uncertainty}

The majority of uncertainty assessment in PIV has focussed on 2D planar measurements. Measurement uncertainty has been investigated a priori using theoretical modelling [31] and Monte Carlo simulation of the measurement chain (e.g. [19] and others). These analyses have helped optimise the various aspects of the measurement chain, with most studies estimating error at approximately $0.1 \mathrm{px}[24]$. Recently, significant attention has been given to the a posteriori quantification of uncertainty in PIV data. A thorough inter-comparison of recently-proposed methods concluded that the a priori estimates of measurement uncertainty do not 
generally apply across different experiments, or even different regions of a single experiment [28], with a posteriori quantification providing a more reliable estimate of overall error.

The novel PIV measurement technique of this study is able to resolve the three components of velocity over the large measurement volume necessitated by the anisotropic island wake flow. A significant advantage of obtaining 3D PIV measurements is the ability to resolve 'out of plane' vertical velocities, which in turn allows the calculation of all nine components of the velocity gradient tensor $(\nabla \mathbf{u})$ using a second-order central differencing scheme. Due to continuity, the divergence should be zero everywhere (i.e. $\nabla \bullet \mathbf{u}=0$ ) in an incompressible flow. Thus, the RMS of the divergence (typically normalised by observed velocity gradients or vorticity) can be evaluated as a measure of experimental error [27, 3]. This approach allows for the measurement error to be assessed a posteriori and aggregates the systematic and random errors associated with the experimental apparatus and PIV algorithm. Previous studies have applied uncertainty analysis [22] and established that error in the velocity gradient can be expressed in terms of the random velocity error $[3,13]$ :

$$
\epsilon(\nabla \bullet \mathbf{u})=\sqrt{\frac{3}{2 \Delta^{2}}} \epsilon(u)
$$

where $\Delta$ is the characteristic length scale of the $3 \mathrm{D}$ vector grid (in pixels). Whilst equation 3 provides an estimate of the absolute error relative to the PIV measurement resolution, we also assess the RMS divergence relative to the magnitude of the measured vorticity; the latter is taken as the $99^{t h}$ percentile of the vorticity vector norm $\left(\|\boldsymbol{\omega}\|_{99}\right.$ where $\left.\boldsymbol{\omega}=\nabla \times \mathbf{u}\right)$ for each experimental condition. The divergence error has a magnitude of $0.03-0.05 \mathrm{px} / \mathrm{px}$ (between $4 \%$ and $11 \%$ of the measured vorticity) with a corresponding velocity error of $0.3-0.4 \mathrm{px}$ (Table 2). This compares well with equivalent tomographic 3D PIV experiments $[27,3]$.

Table 2 Estimated uncertainty in 3D PIV results.

$\begin{array}{lllll}\text { Wake type } & \Delta[\mathrm{px}] & \epsilon(\nabla \bullet \mathbf{u})[\mathrm{px} / \mathrm{px}] & \epsilon(u)[\mathrm{px}] & \nabla \bullet \mathbf{u} /\|\boldsymbol{\omega}\|_{99} \\ \text { Symmetric } & 12 & 0.04 & 0.43 & 0.04 \\ \text { Asymmetric } & 12 & 0.04 & 0.36 & 0.06 \\ \text { Unsteady Bubble } & 12 & 0.03 & 0.32 & 0.08 \\ \text { Vortex Shedding } & 12 & 0.03 & 0.27 & 0.11\end{array}$

\subsection{Data analysis}

In the presentation of the results, spatial averages are denoted by $\langle\ldots\rangle$, time averages by an over-bar (e.g. $\bar{u}$ ). Subscripts are utilised to indicate if a different statistic (other than the mean) is calculated. For example the vertical profile of root mean square velocity is calculated as

$$
\langle\bar{u}\rangle_{r m s}(z)=\sqrt{\frac{1}{N} \sum^{x, y, t} u^{2}}
$$


where $\mathrm{N}$ is the number of velocity samples at each height above the bed.

In an unbounded, laminar oscillatory flow, a Stokes boundary layer will develop with thickness $\delta_{B L}=\frac{3}{4} \sqrt{\pi \nu T}$ [4]. The velocity at the top of the boundary layer, $u_{B L}$, is calculated as the spatially-averaged horizontal velocity $\langle u\rangle$ at a height $z=\delta_{B L}$ for $x / D>2.5$. Due to the presence of the Stokes boundary layer there is a phase shift in the horizontal velocity with distance from the bed. To phase align the results, the time $t=0$ is defined at the first flow zero-crossing of $u_{B L}$. Thus, the phase of each experimental condition is defined as $\phi=t / T \bmod 1$. The sinusoidally-varying flow and fixed control volume of the PIV system results in the observation of two distinct periods. Firstly, a wake 'growth' period when the PIV volume is downstream of the island and the wake vorticity is growing due to flow separation around the island. This is followed by a 'decay' period after flow reversal (with the PIV volume upstream of the island) where the wake vorticity decays and is advected back around the island. The 'growth' period is present for $(0.0<\phi<0.5)$ and the 'decay' period for $(0.5<\phi<1.0)$.

Coherent vortices in the wake can be visualised through plotting iso-surfaces of the $Q$-criterion. The $Q$-criterion defines a vortex by a connected fluid region with a positive second invariant of the velocity gradient tensor $D_{i j}=\partial u_{i} / \partial x_{j}$. The second-order velocity gradient tensor can be decomposed into a symmetric $\left(S_{i j}\right)$ and anti-symmetric part $\left(\Omega_{i j}\right)$ as:

$$
S_{i j}=\frac{1}{2}\left(\frac{\partial u_{i}}{\partial x_{j}}+\frac{\partial u_{j}}{\partial x_{i}}\right)
$$

$$
\Omega_{i j}=\frac{1}{2}\left(\frac{\partial u_{i}}{\partial x_{j}}-\frac{\partial u_{j}}{\partial x_{i}}\right)
$$

with the second invariant $Q$ defined as:

$$
Q=\frac{1}{2}\left(\left\|\Omega_{i j}\right\|-\left\|S_{i j}\right\|\right)
$$

\section{Results}

\subsection{Archetypal island wake flow}

We introduce the results with a schematic presentation of the symmetric wake (Fig. 3). The island generates a region of low pressure downstream; this region of low pressure is often referred to as a wake bubble. Flow separation occurs at the island flanks and the low pressure establishes a recirculation region through entrainment of mass and momentum across the shear layer that extends from the flow separation point (shown in red in on one flank only for clarity - see Fig. 3). The flow approaching the island has a vertically sheared profile, with a vertical structure depending on $\delta^{+}$. The vertical structure of the incoming flow plays an important role in modulating the wake entrainment and wake form. Upwelling occurs in the core of the primary vertical vortices $\left(\omega_{z}\right)$ with flow convergence at the bed and divergence at the free surface. The primary region of downwelling is along the wake centreline associated with two counter-rotating secondary horizontal vortices $\left(\omega_{x}\right)$ with flow convergence at the surface and divergence at the bed. Downwelling occurs in the region of greatest strain and upwelling in the region 
of greatest rotation. The presence of both vertical and horizontal vortices was a ubiquitous feature of all island wake forms observed in this study. However, the co-incidence of up- and downwelling regions with vertical and horizontal vortices (respectively) in the symmetric wake is significantly altered by reducing the relative boundary layer thickness $\left(\delta^{+}\right)$and increasing the relative tidal excursion $(K C)$.

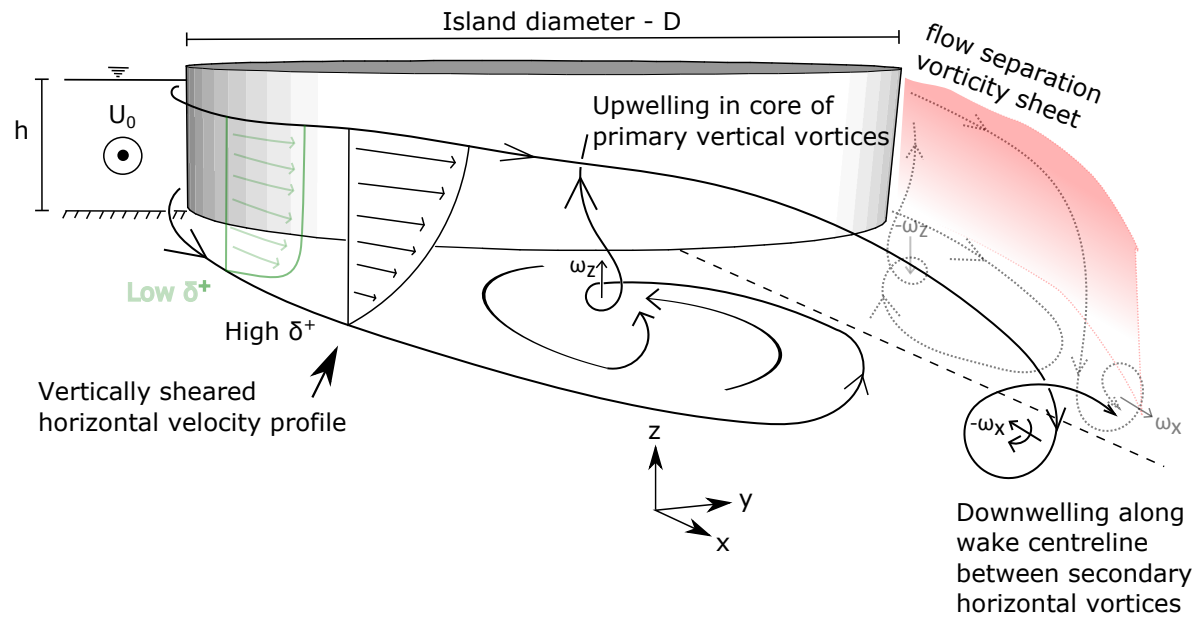

Fig. 3 Schematic diagram of the symmetric wake form at a phase of approximately 0.3 depicting key characteristics of shallow island wake flow. The wake centreline is indicated by the black dashed line, with processes occurring on the right flank of the cylinder presented in gray and dotted. A sheet of vertical vorticity (in red) generated along the separation streamline is only shown on the right flank for clarity, an equivalent (with opposite sign) sheet is also present on the left flank. The strength of the vertical vorticity sheet reduces towards the bed due to the boundary layer. A schematic of the vertical profile of horizontal velocity for the symmetric wake (with high $\delta^{+}$) is shown in black, and for contrast with low $\delta^{+}$in green.

The effect of reducing $\delta^{+}$and increasing $K C$ is presented schematically in plan view to demonstrate the general arrangement of primary and secondary vortices (Fig. 4). The symmetric wake from Fig. 3 is presented in Fig. 4a. Decreasing the relative boundary layer thickness $\left(\delta^{+}\right)$reduces the stabilising effect of friction on the wake, allowing for the onset of asymmetry in the primary vortices and deflection of the secondary vortices (Fig. 4b). Increasing the tidal excursion $(K C)$ for high $\delta^{+}$establishes a wake form where the vertical vortices remain attached during each cycle, but oscillation of the recirculation bubble causes large deflections of the horizontal vortices (Fig. 4c). Finally, increasing the tidal excursion and decreasing the relative boundary layer thickness results in the shedding of primary vortices and intricate arrangements of secondary vortices (braids) that are transported and stretched by the primary vortices and external tidal flow (Fig. 4d). 
(a) Symmetric $\mathrm{KC}=4.5$ $\delta^{+}=0.40$

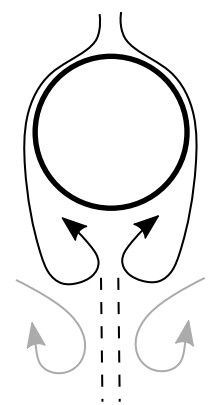

(c) Unsteady Bubble $\mathrm{KC}=24.1$ $\delta^{+}=0.40$

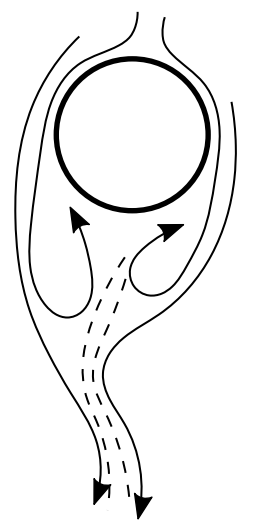

(b) Asymmetric

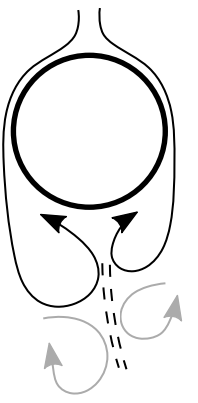

(d) Vortex Shedding $\mathrm{KC}=26.0$ $\delta^{+}=0.26$

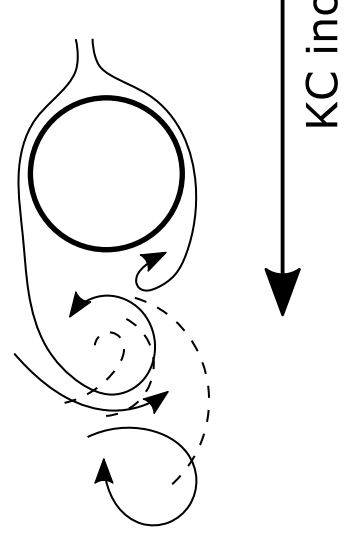

\section{$\delta+$ decreasing}

Fig. 4 Schematic diagram showing a planview of the four wake forms examined in this study at a phase of approximately 0.3. a) Symmetric wake, b) asymmetric wake, c) unsteady bubble wake d) vortex shedding wake. Solid lines are indicative streamlines showing circulation of primary vortices generated on the current cycle. Gray lines show circulation generated on the previous half-cycle pairing with the newly0generated primary vortices. Dashed lines indicate the locations and orientation of secondary horizontal vortices.

\subsection{Island wake structure}

The island wake structure summarised in Section 3.1 is expanded upon for each of the archetypal wake forms. The arrangement of primary and secondary flow structures driving vertical mixing are contrasted through examination of instantaneous vertical velocity at mid-depth (Fig. 5). The spatial distribution of up- and downwelling over the tidal cycle is presented in plots of $99^{t h}$ and $1^{\text {st }}$ percentile of vertical velocity ( $\bar{w}_{99}$ and $\bar{w}_{1}$ respectively) (Fig. 6). The reduced influence of bed friction in island wakes where the relative boundary layer is small $\left(\delta^{+}\right.$small $)$, is demonstrated via the persistence of secondary flow structures into the 'decay' period (Fig. 7). Finally the complex arrangement of upwelling and downwelling flow structures in island wakes where the tidal excursion is long ( $K C$ large) is demonstrated through three-dimensional presentation of the unsteady bubble and vortex shedding wakes (Fig. 8 and Fig. 9 respectively). 

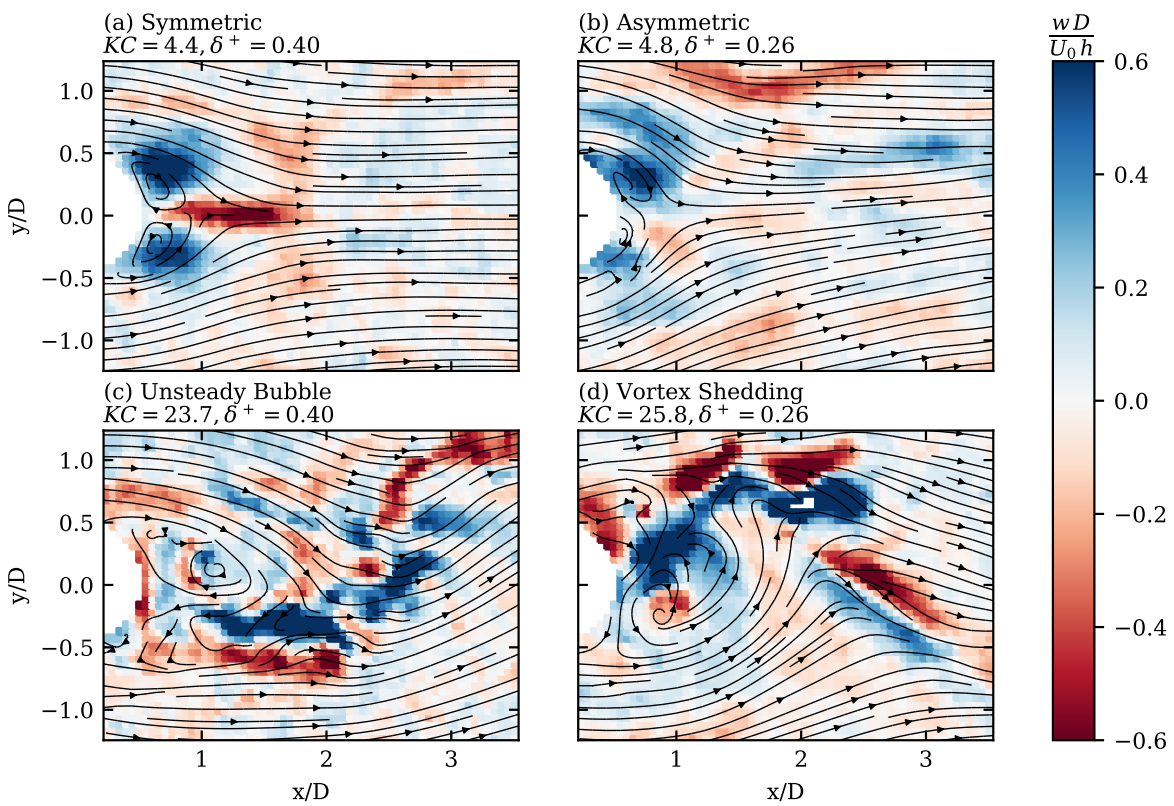

Fig. 5 Instantaneous up- and downwelling measured at mid-depth, with streamlines of instantaneous horizontal velocity at $z=\delta_{B L}$ at phase $t / T=5.35$ for the four experimental conditions (a) symmetric wake (b) asymmetric wake (c) unsteady bubble wake and (d) vortex shedding wake

\subsubsection{Symmetric wake - KC $=4.5, \delta^{+}=0.40$}

The 'simplest' wake form is that of the symmetric wake, where the tidal excursion is small relative to the island size (low $K C$ ), the aspect ratio $h / D$ is small and the bottom boundary layer extends over the full flow depth. In the symmetric wake the flow exhibits a highly repeatable flow structure with little cycle-to-cycle variability (Fig. 12). An example of the instantaneous flow field and vertical velocity is shown for $t / T=5.35$ in Fig. 5a. Upwelling is concentrated in the centre of the primary vertical vortices and downwelling in the region of strain along the wake centreline. Along the separation streamline around the flanks of the wake a weak downwelling region is present. The spatial distribution of up- and downwelling in this regime is consistent with analytical modelling of a classical dipolar vortex [18]. Downwelling is also present on the upstream side of the cylinder during the 'decay' phase due to the formation of a horseshoe vortex (Fig. 6b). Upwelling and downwelling regions are highly localised and consistent from cycle to cycle, as demonstrated by the spatial distribution of peak of up- and downwelling velocity presented in Fig. 6a and $6 \mathrm{~b}$, respectively.

\subsubsection{Asymmetric wake - $K C=4.7, \delta^{+}=0.26$}

The reduction of the influence of bed friction (decreasing $\delta^{+}$) results in the development of asymmetry in the wake, even when the relative tidal excursion is small 

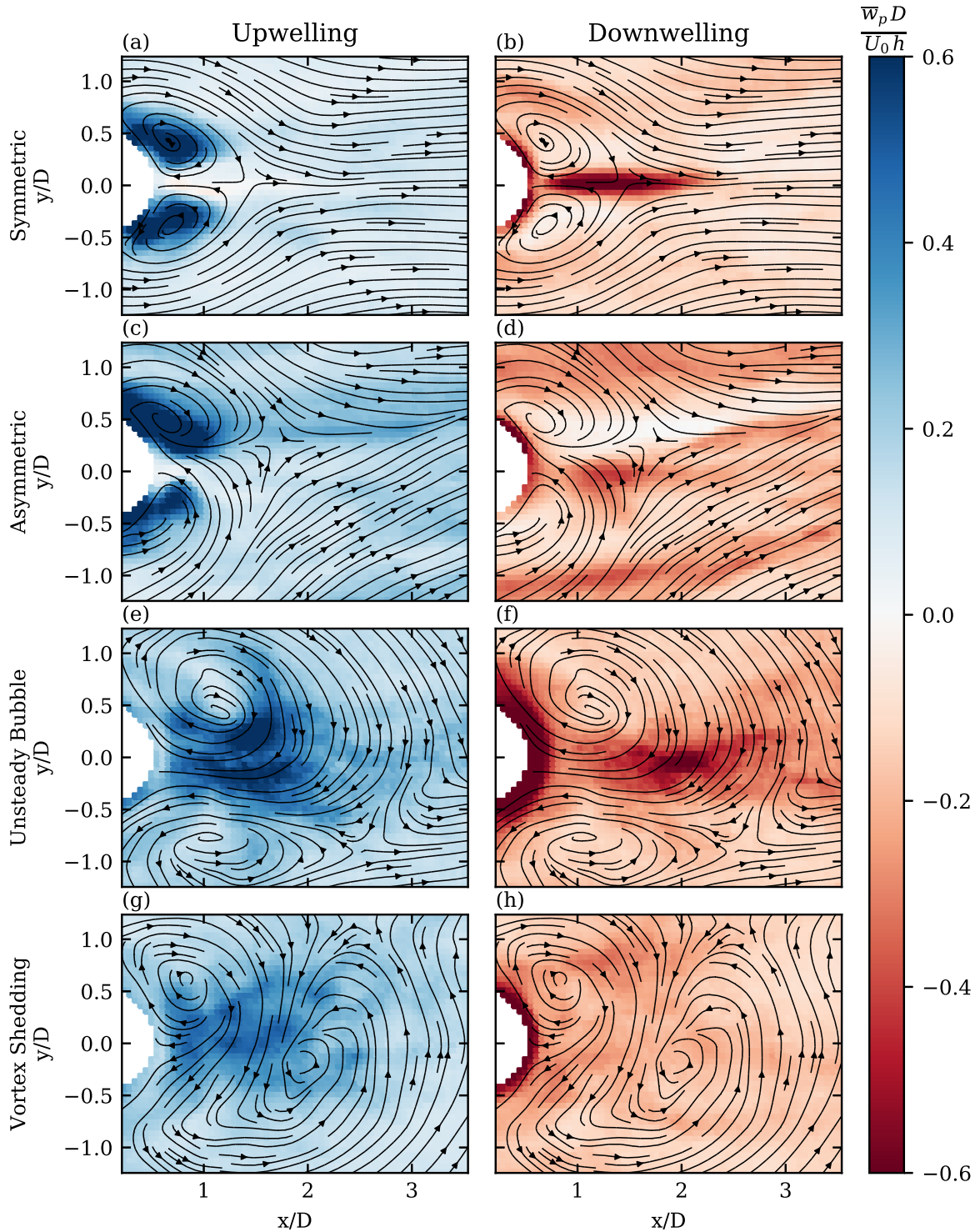

Fig. 6 Spatial distribution in the four wake forms of (a,c,e,g) upwelling magnitude at middepth, indicated by the $95^{\text {th }}$ percentile of vertical velocity and (b,d,f,h) downwelling magnitude, indicated by the $5^{\text {th }}$ percentile of vertical velocity. Streamlines of phase-averaged horizontal velocity at the end of the 'growth' phase are shown at $z=\delta_{B L}$.

(low $K C$, Fig. 5b). In studies of unbounded cylinders asymmetry commences at $K C \approx 4[33]$. In the shallow island wake with $K C=4.5$ and $\delta^{+}=0.40$ asymmetry is suppressed, however at $\delta^{+}=0.26$ asymmetry is present. This implies that a transition in wake form occurs between $0.26<\delta^{+}<0.40$ for low $K C$ due to the reduction in the bottom boundary layer thickness. The up- and downwelling is 
sustained throughout the tidal cycle as demonstrated by the time variation of the vertical velocity (Fig. 12). The dominant region for upwelling remains associated with the primary wake vertical vortices (Fig. 6c). However, the downwelling is no longer only associated with the region of strain between the primary vortices. Three bands of downwelling are present, extending from the flanks and centreline of the island (Fig. 6d). The flow structures that contribute to this are presented in Fig. 7a, which shows the instantaneous up- and downwelling at mid-depth and streamlines of horizontal velocity at $z=\delta_{B L}$ at the end of the 'decay' phase $(t / T=0.0)$. Significant vertical transport is associated with $x$-aligned flow structures that exhibit a decay time scale considerably longer than the primary vortices, as evidenced by their persistence through the 'decay' period. It is these longitudinal flow structures that sustain the up- and downwelling flow throughout the tidal cycle for this experimental condition. Signatures of these flow structures are present for other experimental conditions at the end of the 'decay' phase, however their strength (normalised by $U_{0}$ ) is reduced considerably compared to other flow features (i.e. the horseshoe vortex).
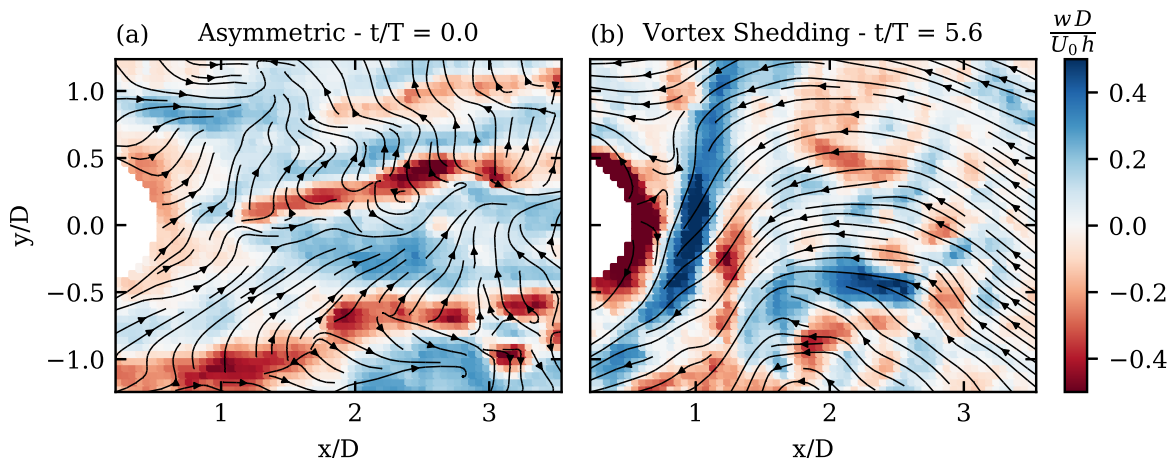

Fig. 7 The instantaneous fields of up- and downwelling measured at mid-depth for (a) the asymmetric wake at the end of the 'decay' phase $(t / T=0.0)$ and $(b)$ the vortex shedding wake at the beginning of the 'decay' phase $(t / T=6.1)$. Streamlines are shown at $z=\delta_{B L}$. In the asymmetric wake, $x$-aligned vortices, whose longitudinal extent is significantly greater than the tidal excursion, contribute significantly to up- and downwelling. In the vortex shedding wake, a $y$-aligned vortex interacts with the island and drives strong downwelling (also seen later in Fig. 12b).

\subsubsection{Unsteady bubble wake - $K C=24.1, \delta^{+}=0.40$}

Increasing the tidal excursion relative to the island size in the case of high friction results in the establishment of the 'unsteady bubble' wake, as observed in steady shallow flows [9]. Increasing cycle-to-cycle variability in up- and downwelling is present (Fig. 12). As a consequence of the strong advection of the up- and downwelling flow structures, the envelopes of vertical velocity presented in Fig. 6e and 6f are more spatially diffuse than in the symmetric and asymmetric wake forms. The dominant region of upwelling is shifted away from the vortex centres due to advection in the primary vortices. Additional upwelling regions are established 
coincident with downwelling regions in secondary horizontal vortices that extend from the centreline of the oscillating recirculation bubble (Fig. 5c). This is seen in the three-dimensional presentation of the unsteady bubble wake in Fig. 8) Fig. 8a presents streamlines seeded in a uniform grid at the bed and coloured by their elevation. Upwelling in the primary vortices is advected around the strong, swirling flow. Iso-surfaces for $Q=0.2$ are presented in Fig. 8b. A strong secondary horizontal vortex is observed, extending from the near wake, with considerable associated up- and downwelling flows (Fig. 8c).

\subsubsection{Vortex shedding wake $-K C=26.3, \delta^{+}=0.26$}

When the relative influence of bed friction is reduced (decreasing $\delta^{+}$) and the tidal excursion is sufficiently long compared to the size of the island (i.e. large $K C$ ), the vortex shedding process is observed (Fig. 6g). At the onset of vortex shedding, the developing vortex on one side draws the separation shear layer of opposite sign across the wake. The cross-wake supply of oppositely signed vorticity cuts off the supply to the growing vortex which leads to detachment from the shear layer [27]. In contrast to the unbounded cylinder wake, the no slip bottom boundary in shallow island wakes reduces the lateral shear in the bottom boundary layer, and alters the vertical distribution of momentum that is entrained into the wake. The transition in wake form between $\delta^{+}=0.26$ and $\delta^{+}=0.4$ for given $K C$ (see the streamlines in Figs. $6 \mathrm{~g}$ and $6 \mathrm{e}$ ) implies that the increasing boundary layer thickness attenuates the processes that lead to detachment of the primary vortices.

Vortex shedding wakes are comprised of an intricate tapestry of primary and secondary vortices (Fig. 5d). Advection of vorticity is a dominant process and upand downwelling flow structures are subjected to significant transport, tilting and stretching. As the primary vortices grow and shed, numerous secondary horizontal vortices form, are stretched between the primary vortices and, as in the 'unsteady bubble' wake, can extend over significant distances (Fig. 5d). Upwelling is concentrated in the centre of the primary vortices and these secondary horizontal vortices. Similarly to the asymmetric wake (which also has low $\delta^{+}$), the horizontal secondary vortices can persist into the 'decay' phase. This is shown in Fig. 7b, where a horizontal vortex of considerable extent has been turned by the primary vortices and advects back past the island at $t / T=6.1$. Vortex stretching around the island induces a significant downwelling peak at $t / T=6.1$ (see Fig. $12 \mathrm{~b}$ ).

The shedding and advection of vortical flow structures produces a spatiallydiffuse field of up- and downwelling. Upwelling is strongest in the primary wake generation region $(x / D<2$ and $-0.5<y / D<0.5$, Fig. $6 \mathrm{~g})$. The spatial distribution of downwelling is considerably more diffuse as downwelling is associated with the horizontal secondary vortices that are subject to significant advection (Fig. 6h). This complexity of the flow field is demonstrated through visualisation of the instantaneous flow field at phase $t / T=0.35$ (Fig. 9). The primary vortex is clearly seen in the streamlines seeded at the bed (Fig. 9a). Numerous horizontal secondary vortices extend between the primary vortex close to the cylinder and a detached primary vortex passing out of frame (Fig. 9b). Concentrated, coherent regions of up- and downwelling are associated with the secondary vortices, with peak magnitudes exceeding $30 \%$ of $U_{0}$ at this instant (Fig. 9c). 


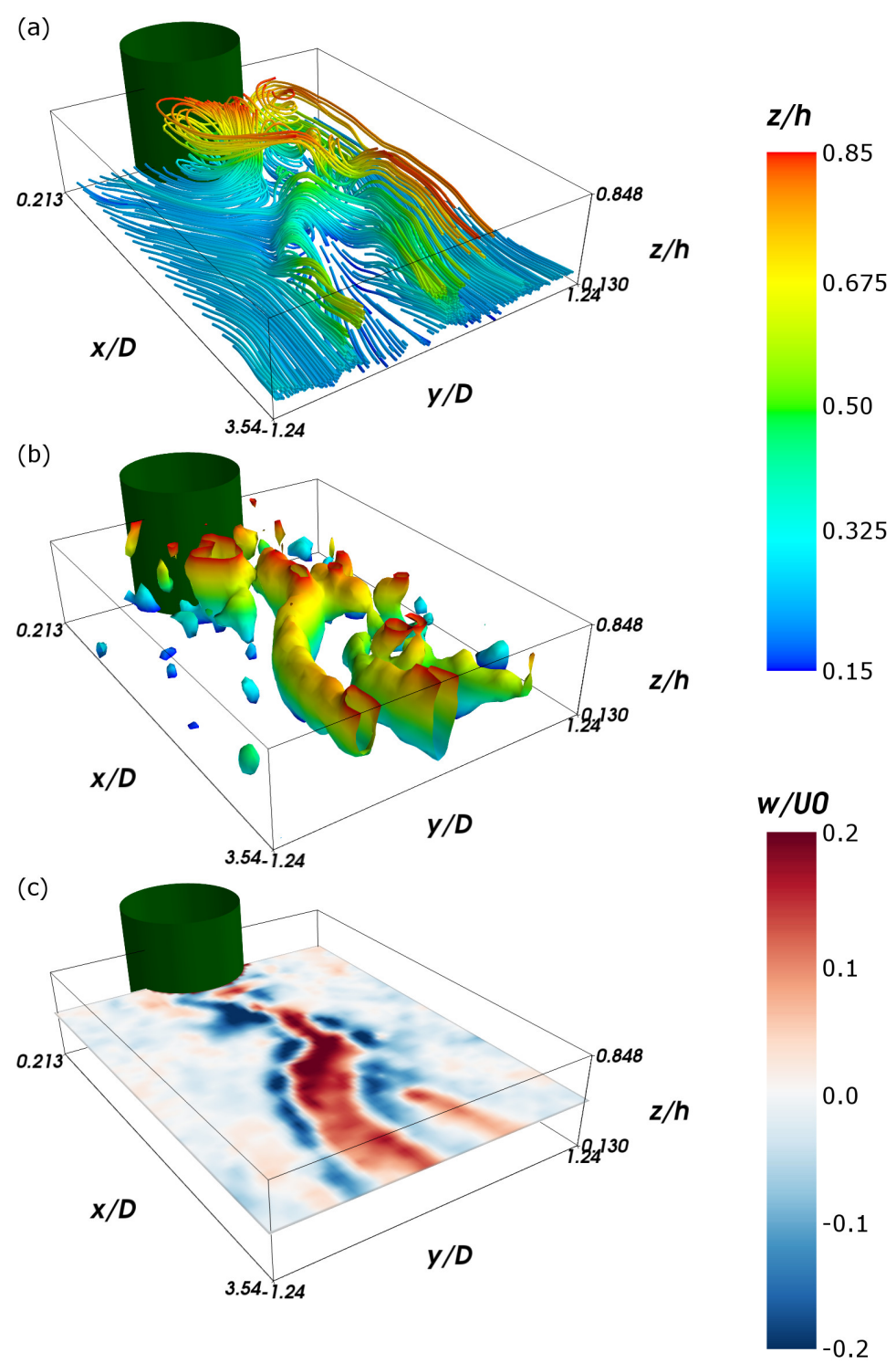

Fig. 8 Visualisation of the instantaneous flow field in the 'unsteady bubble' wake at $t / T=4.2$ (a) Streamlines seeded close to the bed and coloured by elevation, (b) iso-surfaces of the $Q$ criterion at $Q=0.2$ and (c) vertical velocity at mid-depth. Note: the z-axis in the plots is stretched by $D / h=2.3$

\subsection{External tidal flow}

414 The characteristic forcing velocity scale $\left(U_{0}\right)$ is calculated as the average magnitude of the negative peaks in $u_{B L}$ over 8 cycles (i.e. the peak velocity at the top of the 


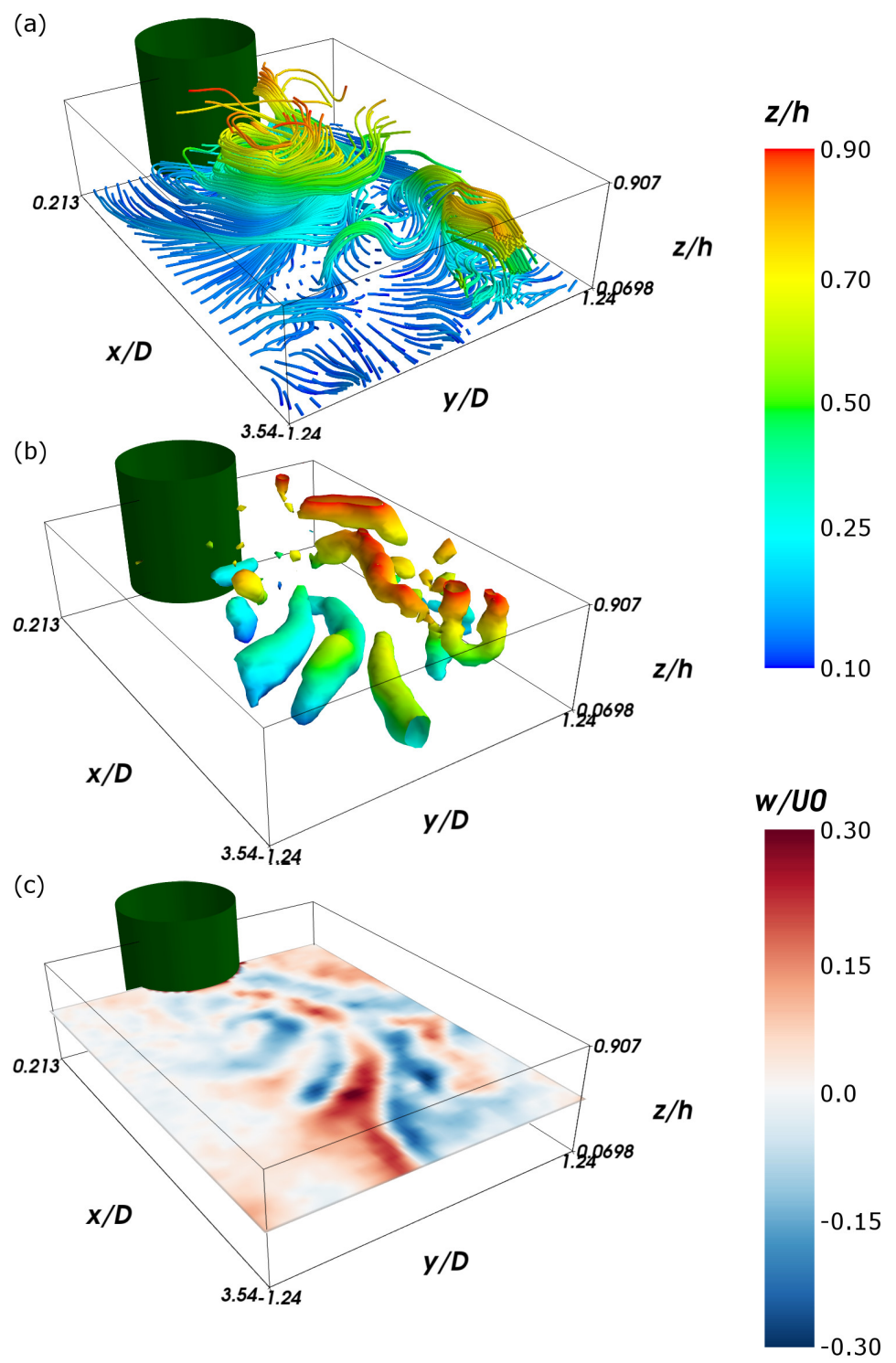

Fig. 9 Visualisation of the instantaneous flow field in the 'vortex shedding' wake at $t / T=0.35$ (a) Streamlines seeded close to the bed and coloured by elevation, (b) iso-surfaces of the $Q$ criterion at $Q=0.2$ and (c) vertical velocity at mid-depth. Note: the z-axis in the plots is stretched by $D / h=4.3$

boundary layer approaching the island at a distance $x / D>2.5$ upstream). Good consistency in the time series of $u_{B L} / U_{0}$ is exhibited across the wide range of forcing conditions examined (Fig. 10a). The peak magnitude of $u_{B L} / U_{0}$ in the 
'growth' period is greater than during the 'decay' period (Fig. 10a). This implies that the island is capturing momentum due to convection into the near wake. This effect is reduced in the vortex shedding wake where the peak in $u_{B L}$ during the 'growth' period is roughly equal to $U_{0}$ due to shedding vortices liberating momentum from the near wake region.

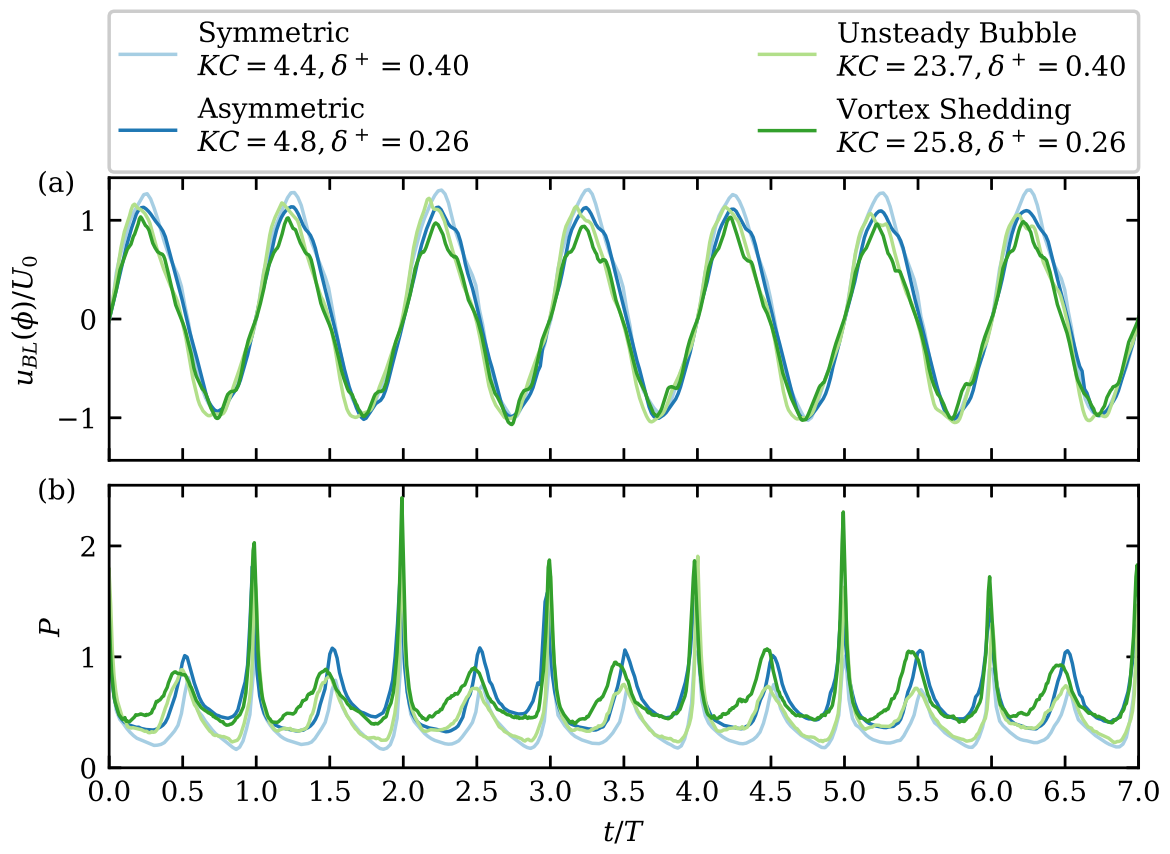

Fig. 10 Overview of the time variability of the island wake flow field. The phase of each cycle is defined as $\phi=t / T \bmod 1$. For the 'growth' period $(0.0<\phi<0.5)$, the measurement volume encompasses the developing wake. During the 'decay' period $(0.5<\phi<1.0)$, the cylinder is at the downstream edge of the measurement volume. (a) Velocity $u_{B L}$ measured at a height of $z=\delta_{B L}$ at distances $x / D>2.5$ from the cylinder for each experimental condition in Table 1. (b) Time variation of the dimensionless parameter $P$ that quantifies the deviation of the vertical profile of horizontal velocity from a Poiseuille-like profile.

\subsection{Vertical structure of the flow}

Examination of the vertical profiles of the three velocity components can be used to identify the importance of vertical transport of momentum to the bed in the wake vortices and the external tidal flow. Previous studies have demonstrated that shallow flows dominated by vertical diffusion of momentum to the bed have a Poiseuille-like profile (with vertical variation of the form $\sin \pi z / 2 h$ ) due to Rayleigh damping [26]. A dimensionless parameter that characterises the vertical structure 
of the horizontal flow field is

$$
P(t ; h)=\sqrt{\frac{\left\langle\left\|\mathbf{u}_{H}-\mathbf{u}_{H}^{p}\right\|^{2}\right\rangle}{\left\langle\left\|\mathbf{u}_{H}\right\|\right\rangle}},
$$

where $\mathbf{u}_{H}(x, y, z, t)$ denotes the horizontal components of the measured 3D velocity field and $\mathbf{u}_{H}^{p}(x, y, z, t)=\mathbf{u}_{H}(x, y, z=h, t) \sin \pi z / 2 h$, is the Poiseuille-like profile [2]. If $P \approx 0$, the flow is very close to a Poiseuille-like flow and can be said to be quasi-two-dimensional. By spatially averaging $P$ over the measurement volume, a single time-varying parameter can be used to compare the vastly different island wake flows in our study (Fig. 10b). The Stokes boundary layer, island boundary layer and island wake result in significant vertical variation and (as expected) at no time can any of the flow conditions be considered quasi-two-dimensional.

The island wake forms during the 'growth' period between $0.0<\phi<0.5$. Both the relative magnitude of the horizontal flow (quantified by $K C$ ) and the degree of bed friction (quantified by $\delta^{+}$) influence the profile of horizontal velocity. The three-dimensionality of the vortex shedding wake increases throughout the 'growth' period, in contrast, three-dimensionality decreases in the symmetric wake despite the growing wake vortices (Fig. 10b). The asymmetric wake and unsteady bubble wake obtain a more or less constant value of $P$ throughout this period despite significant differences in the excursion length of the external flow $(K C=4.8$ and 24.1 respectively).

During the 'decay' period $(0.5<\phi<1.0)$ all flows exhibit a decay towards a Poiseuille-like profile, with a minimum value of $P$ that decreases with increasing $\delta^{+}$, independent of $K C$ (Fig. 10b). This highlights the importance of $\delta^{+}$in describing the fundamental influence of bed friction, independent of the excursion length of the horizontal flow and decaying wake. In a laminar Stokes boundary layer, flow reversal at the bed leads flow reversal outside the boundary layer by approximately $45^{\circ}(0.125 \phi)$ and this behaviour generates a rapid increase in $P$ between $0.9<\phi<1.0$ in all flows (Fig. 10b).

For fixed $K C$ there is clearly a regime change between $\delta^{+}=0.26$ and $\delta^{+}=$ 0.40 . This regime change due to the increasing influence of bed friction in the forcing flow is demonstrated in the spatially-averaged vertical profile of $\langle\bar{u}\rangle_{r m s}$ during the 'decay' period (see Fig. 11d). There is a reasonable collapse of the vertical profiles of $\langle\bar{u}\rangle_{r m s}$ for each value of $\delta^{+}$, largely independent of $K C$. The flows with $\delta^{+}=0.26$ exhibit a defined boundary layer of height approximately equal to $\delta_{B L}$. In contrast, those with $\delta^{+}=0.40$ exhibit vertical shear over the entire flow depth (Fig. 11d). During the 'growth' period, $\delta^{+}$again governs the overall form of the vertical profile, however for low $K C$ a marked increase in $\langle\bar{u}\rangle_{r m s}$ (compared to the 'decay' period) is observed due to trapping of momentum in the wake (Fig. 11a). This increase in momentum in the near wake implies a convective acceleration associated with the pairing of newly-generated wake vortices with vorticity from the previous half cycle, which is also exhibited in the enhanced peak $u_{B L}$ values in the 'growth' period (Fig. 10d).

The magnitude of $\langle\bar{v}\rangle_{r m s}$ is indicative of the swirling flow generated in the island wake. A clear boundary layer in $\langle\bar{v}\rangle_{r m s}$ is present for all cases during the 'growth' period (Fig. 11b). For low $K C$ the boundary layer thickness exhibited during the 'growth' period persists through the 'decay' period. In contrast for large $K C$ a near vertical (inviscid) profile in $\langle\bar{v}\rangle_{r m s}$ is present during the 'decay' 


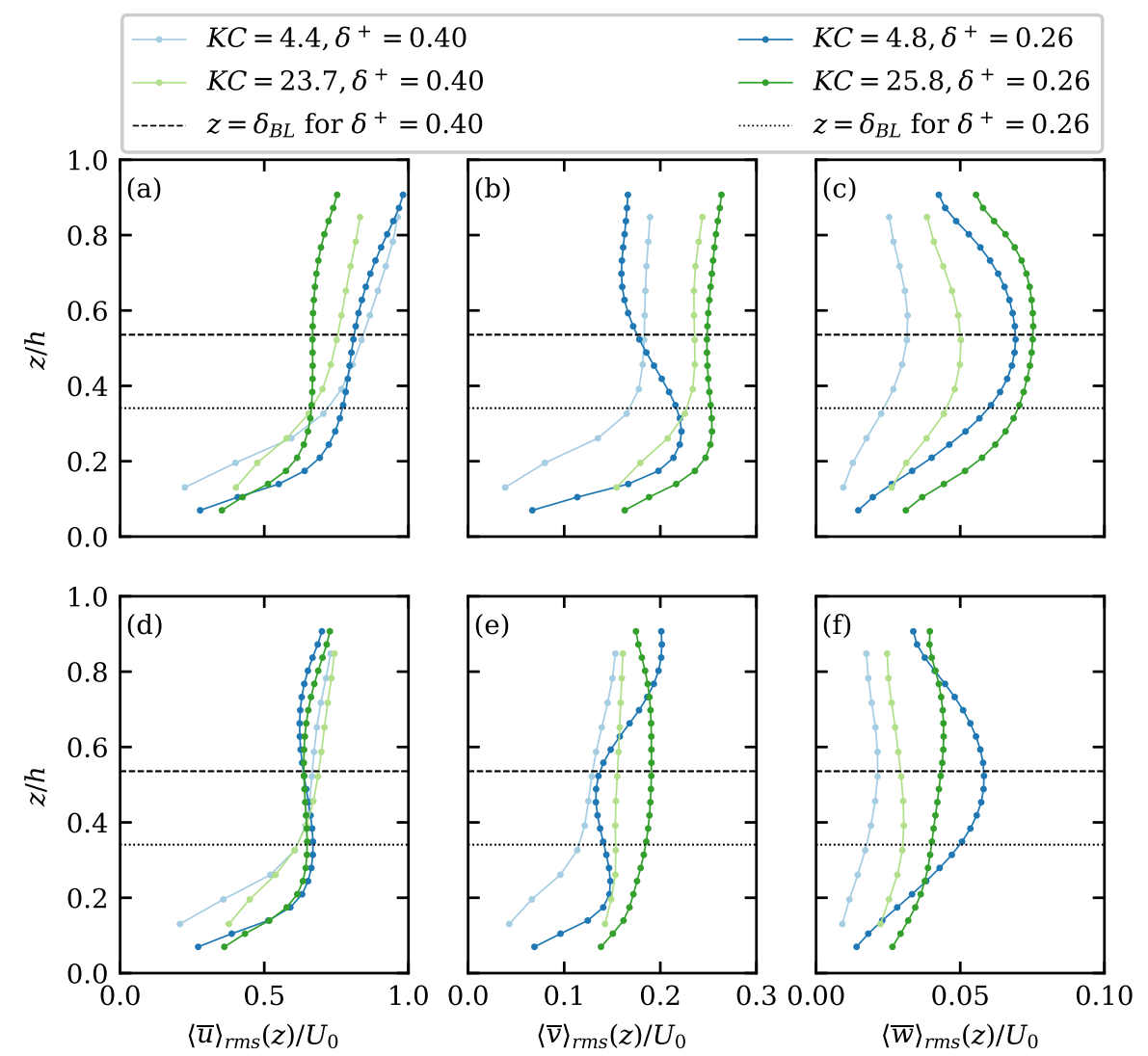

Fig. 11 Overview of vertical structure of the unsteady island wake flow. The time average is denoted by an over-bar, spatial average by $\langle\ldots\rangle$ and the subscript rms indicates the root mean square such that each point in the field is squared and then averaged over space and time at each height $z / h$. (a-c) Vertical profile of $\langle\bar{u}\rangle_{r m s},\langle\bar{v}\rangle_{r m s}$ and $\langle\bar{w}\rangle_{r m s}$ during the 'growth' period. (d-f) Vertical profiles of $\langle\bar{u}\rangle_{r m s},\langle\bar{v}\rangle_{r m s}$ and $\langle\bar{w}\rangle_{r m s}$ during the 'decay' period.

period (Fig. 11e). The boundary layer thickness during the 'growth' period is a function of $\delta^{+}$rather than $K C$. This demonstrates that the vertical structure of the swirling flow in the shallow island wake is a function of the external scale of $\delta^{+}$rather than of the intrinsic time scale of vortex revolution (which scales on $\left.D / U_{0}\right)$. This highlights a fundamental difference between an island wake vortex, where the circulation is established through entrainment across a free shear layer, and an analytical or numerical vortex where the circulation is established through some initial azimuthal velocity profile within the vortex.

The up- and downwelling velocity is largest around mid-depth (Fig. 11c). The magnitude of vertical velocity is greatest during the 'growth' period and indicates a dependence on the aspect ratio $h / D$ and $K C$ (Fig. 11c) - see Section 3.5. Of particular note is the strong persistence of $\langle\bar{w}\rangle_{r m s}$ during the 'decay' period for the $K C=4.8$ and $\delta^{+}=0.26$ condition (Fig. 11f). As will be demonstrated later, this is due to the establishment of $x$-aligned recirculation cells, a feature that, 
whilst present for all experimental conditions, are a dominant feature when the tidal excursion is small compared to the island scale (low $K C$ ) and the flow deep compared to the boundary layer thickness (low $\left.\delta^{+}\right)$.

\subsection{Vertical circulation in island wakes}

The strength of the upwelling velocity is examined through calculation of the $99^{\text {th }}$ percentile of each instantaneous 3D field $\langle w\rangle_{99}$ (Fig. 12). Upwelling commences in the 'growth' period, soon after flow reversal, for all flow conditions. The unsteady bubble and vortex shedding wakes exhibit significantly greater cycle-to-cycle variability than the symmetric and asymmetric wakes. Upwelling in the unsteady bubble wake generally peaks earlier than the other wake forms and the magnitude of upwelling in the asymmetric wake has significantly greater persistence through the decay phase (Fig. 12a).

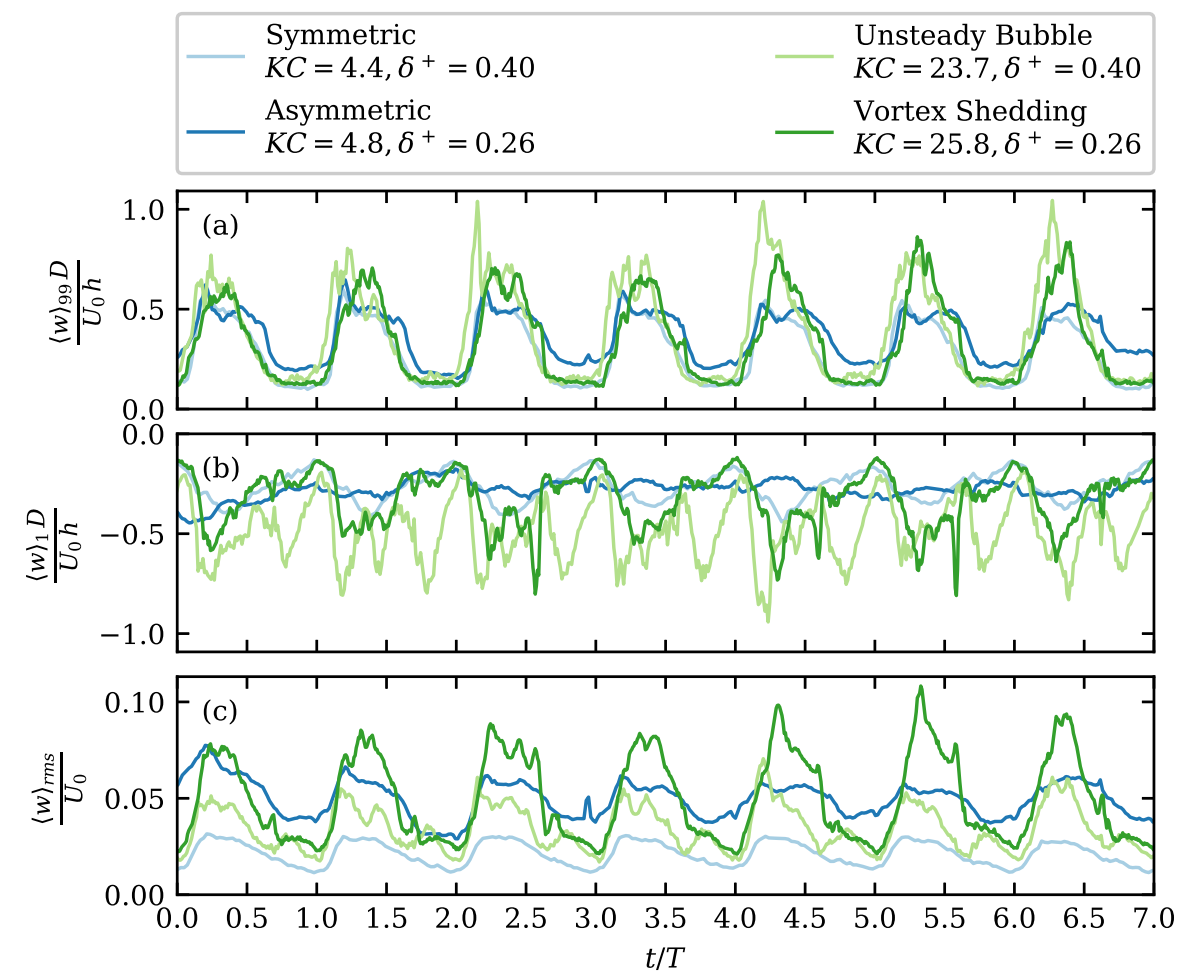

Fig. 12 Time variation of (a) the upwelling strength indicated by the $99^{t h}$ percentile of vertical velocity $\langle w\rangle_{99} / U_{0}$ (b) the downwelling strength indicated by the $1^{\text {st }}$ percentile of vertical velocity $\langle w\rangle_{1} / U_{0}$ and (c) the root-mean-square vertical velocity for the four wake forms.

It is commonly assumed that the vertical velocity is much smaller than the horizontal velocity due to the vertically-constrained shallow flow. From continuity, 
we would expect the upwelling velocity to scale according to $\langle w\rangle_{99} / U_{0} \sim h / D$. The relative upwelling strength does scale primarily on the aspect ratio $h / D$, with a weak dynamic dependence on the relative tidal length given by $K C$ (Fig. 12a).

The up- and downwelling velocities in island wakes are of the same order of magnitude (Fig. 12b). The upwelling magnitude exhibits a single prolonged period of upwelling throughout the 'growth' period that peaks around $\phi=0.3$. Downwelling is distributed through both the 'growth' and 'decay' periods in the unsteady bubble wake due to the formation of a strong downwelling horseshoe vortex during the 'decay' period (Fig. 12b and 6f). Despite $\delta^{+}$being fundamental in establishing the vertical profile of horizontal velocity, it has only a minor influence on the strength of the relative upwelling velocity compared to the aspect ratio $h / D$. However, by controlling the vertical profile of horizontal velocities, both $\delta^{+}$and $K C$ determine the wake form, and in turn, the spatial and temporal distribution of upwelling and downwelling.

The root-mean-square vertical velocity (normalised by $U_{0}$ ) gives an indication of the average vertical transport across the measurement volume at each time, with values ranging from $3-10 \%$ of $U_{0}$ (Fig. 12c). Further, the $99^{\text {th }}$ percentile of vertical velocity is up to 3 - 4 times greater than the rms values (i.e. up to $40 \%$ of $\left.U_{0}\right)$. The measured vertical velocity is thus at least an order of magnitude greater than previous estimates of island wake upwelling velocities [36].

\section{Discussion}

The translation of laboratory-scale experiments with idealised circular islands to the field scale requires careful consideration. The PIV technique utilises an interrogation volume that has a finite spatial scale. Sub-interrogation-volume shear and rotation is not resolved by PIV measurements. The PIV algorithm employed in this study applied a multi-gridded window deformation technique that improves robustness and accuracy in highly sheared flows such as boundary layers and vortices [24]. The vertical profile of rms vorticity and measurement divergence is shown in Fig. 13. The measurement divergence (Fig. 13b) increases somewhat in the strongly sheared bottom boundary layer (Fig. 13a). The measurement divergence also increases near the free surface (Fig. 13b), however it is possible that the measurements resolved physical divergence associated with free surface perturbation that was visually observed to be induced by the primary and secondary vortices. The use of the measurement divergence to estimate the a posteriori uncertainty of 3D PIV data in free surface flows is potentially somewhat conservative. In addition, due to the finite scale of the interrogation volumes, the measured peaks in up- and downwelling velocity are potentially underestimated due to the small-scale features being averaged over the interrogation volume. Despite these second-order measurement issues, we have shown incredibly strong vertical velocities, with coherent and complex wake structure that supports the general framework for island wake description developed in the study.

Localised peaks in up- and downwelling velocity were observed to be up to $15-40 \%$ of the peak tidal flow velocity, a significant departure from a purely horizontal flow field. This brings into question the ability of 3D modelling studies that apply the shallow water equations with a hydrostatic assumption to resolve vertical transport processes in topographically-complex coastal regions. In this 


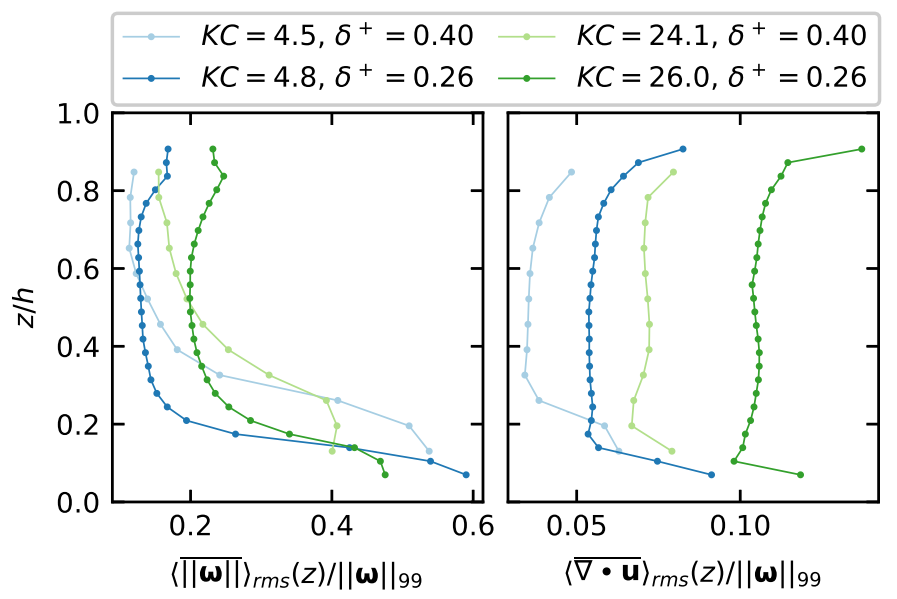

Fig. 13 Vertical profile of (a) root mean square vorticity and (b) measurement divergence for the four experimental conditions.

study we utilised the idealised island geometry of a circular cylinder with vertical side walls, where as in the field, islands tend to have sloping sides. The vertical side walls can cause separation of flow from the bed and the generation of a horseshoe vortex system, which was observed in these experiments during the peak flow of the 'decay' phase for the large $K C$ conditions. However, consistent with conclusions of a detailed 2D PIV study of a vortex shedding wake in steady flow with $h / D=0.5$ [1], the horseshoe vortex system was observed to have little influence on the circulation of the wake region as it is located outside the separation streamline of the lateral shear layer. It is acknowledged that for sloping sides, the details of the lateral shear layer at the point of flow separation will be quite different due to the orientation of the boundary. However, studies of the wake of conical shaped islands in shallow, steady flow have demonstrated that the wake forms are broadly similar to those of cylindrical islands for a given value of $S$ based on the diameter at mid-depth [20]. Furthermore, studies of shallow, electromagneticallyforced vortical flows exhibit characteristically similar secondary flow structures, in the absence of flow separation from a boundary [2]. Therefore, the highly threedimensional structure of the wake region observed in the laboratory is likely also present in the field (for similar $h / D$ ), as evidenced by complex ocean surface signatures of, for example, suspended particles.

A challenge in transference of these results to the field scale is in the estimation of a vertical eddy viscosity in the bottom boundary layer that dictates the boundary layer thickness (i.e. $\delta^{+}$). It is clear that the relative boundary layer thickness, and therefore the presence or absence of an inviscid upper layer in the flow, is a key parameter controlling the growth of instability in the wake.

One-dimensional models of the water column, based on diffusive vertical fluxes of nutrients to the euphotic zone, are commonly applied to study biological processes. This study suggests that vertical nutrient fluxes in island wakes may be driven by advective, rather than diffusive, processes. Thus, nutrient fluxes and the residence times of biological species in the euphotic zone may be poorly repre- 
sented by a 1D model, limiting their applicability in estimating productivity in these systems.

\section{Conclusion}

The results presented in this study clearly demonstrate significant three-dimensionality in shallow island wakes. The normalised upwelling and downwelling velocities across the four wakes were all of the same order of magnitude, and the ratio of the upwelling velocity to the external tidal velocity scaled primarily with $h / D$. Upwelling occurs predominantly in the core of primary vertical vortices, with downwelling in long secondary horizontal vortices. Three-dimensionality is an inherent feature of shallow island wakes, with horizontal vortices playing a central role in vertical mixing. The relative boundary layer thickness $\left(\delta^{+}\right)$plays an important role in establishing the overall wake form and, in turn, the spatial distribution of vertical mixing. The relative tidal excursion (as indicated by $K C$ ) also governs the wake form and, in conjunction with the aspect ratio $(h / D)$, determines the magnitude of vertical circulation.

Acknowledgements P. M. Branson gratefully acknowledges the support of a University Club of Western Australia Research Travel Scholarship to attend the 4th International Symposium on Shallow Flows. The authors acknowledge numerous insightful discussions with E. J. Hopfinger during his visit to UWA on a Gledden Visiting Fellowship. This work was supported by resources provided by The Pawsey Supercomputing Centre with funding from the Australian Government and the Government of Western Australia. We acknowledge the use of imagery provided by services from the Global Imagery Browse Services (GIBS), operated by the NASA/Goddard Space Flight Center Earth Science Data and Information System (ESDIS) project.

\section{References}

1. Akilli H, Rockwell D (2002) Vortex formation from a cylinder in shallow water. Phys Fluids 14(9):2957-2967, DOI 10.1063/1.1483307

2. Akkermans RAD, Kamp LPJ, Clercx HJH, van Heijst GJF (2008) Intrinsic three-dimensionality in electromagnetically driven shallow flows. Europhys Lett 83(2):24001, DOI 10.1209/0295-5075/83/24001

3. Atkinson C, Coudert S, Foucaut JM, Stanislas M, Soria J (2011) The accuracy of tomographic particle image velocimetry for measurements of a turbulent boundary layer. Exp Fluids 50(4):1031-1056, DOI 10.1007/s00348-010-1004-z

4. Batchelor G (1967) An introduction to fluid dynamics. Cambridge mathematical library, Cambridge University Press, DOI 10.1017/cbo9780511800955

5. Belden J (2013) Calibration of multi-camera systems with refractive interfaces. Exp Fluids 54(2):1-18, DOI 10.1007/s00348-013-1463-0

6. Belden J, Truscott TT, Axiak MC, Techet AH (2010) Three-dimensional synthetic aperture particle image velocimetry. Meas Sci Technol 21(12):125403, DOI 10.1088/0957-0233/21/12/125403

7. Branson P, Ghisalberti M, Ivey G (2016) Time resolved 3d3c measurements of shallow-water island wakes. In: 20th Australasian Fluid Mechanics Conference

8. Branson P, Ghisalberi M, Ivey G, Hopfinger E (2018) Scaling of upwelling and secondary circulation in shallow island wakes. J Fluid Mech to appear 
9. Chen D, Jirka GH (1995) Experimental study of plane turbulent wakes in a shallow water layer. Fluid Dyn Res 16(1):11 - 41, DOI 10.1016/0169-5983(95) 00053-g

10. Chopra K, Hubert L (1965) Mesoscale eddies in wake of islands. J Atmos Sci 22(6):652-657, DOI 10.1175/1520-0469(1965)022〈0652:meiwoi $\rangle 2.0 . c o ; 2$

11. Chu V, Wu J, Khayat R (1983) Stability of turbulent shear flows in shallow channel. In: Proceeding of the 20th Congress of IAHR, Moscow, vol 3, pp 128-133

12. Duran-Matute M, Kamp LPJ, Trieling RR, van Heijst GJF (2012) Regimes of two-dimensionality of decaying shallow axisymmetric swirl flows with background rotation. J Fluid Mech 691:214

13. Earl TA, Paetzold J, Cochard S (2013) Tomographic piv measurements of turbulent fountains with refraction index matching. Journal of Flow Visualization and Image Processing 20(3), DOI 10.1615/jflowvisimageproc.2014011727

14. Hoitink A (2004) Tidally-induced clouds of suspended sediment connected to shallow-water coral reefs. Mar Geol 208(1):13 - 31, DOI https://doi.org/10. 1016/j.margeo.2004.04.021

15. Ingram RG, Chu VH (1987) Flow around islands in rupert bay: An investigation of the bottom friction effect. J Geophys Res 92(C13):14521-14533, DOI 10.1029/jc092ic13p14521

16. Jenner K, Jenner M, McCabe K (2001) Geographical and temporal movements of humpback whales in western australian waters. APPEA Journal 38(1):692707, DOI 10.1071/aj00044

17. Johnston DW, Read AJ (2007) Flow-field observations of a tidally driven island wake used by marine mammals in the bay of fundy, canada. Fish Oceanogr 16(5):422-435, DOI 10.1111/j.1365-2419.2007.00444.x

18. Kamp LPJ (2012) Strain-vorticity induced secondary motion in shallow flows. Phys Fluids 24(2):023601, DOI 10.1063/1.3682097

19. Keane RD, Adrian RJ (1992) Theory of cross-correlation analysis of piv images. Appl Sci Res 49(3):191-215, DOI 10.1007/bf00384623

20. Lloyd PM, Stansby PK (1997) Shallow-water flow around model conical islands of small side slope. I: Surface piercing. J Hydraul Eng 123(12):1057-1067, DOI 10.1061/(ASCE)0733-9429(1997)123:12(1057)

21. Lloyd PM, Stansby PK, Chen D (2001) Wake formation around islands in oscillatory laminar shallow-water flows. part 1. experimental investigation. J Fluid Mech 429:217-238, DOI 10.1017/s0022112000002822

22. Moffat RJ (1988) Describing the uncertainties in experimental results. Exp Therm Fluid Sci 1(1):3-17, DOI 10.1016/0894-1777(88)90043-x

23. Pattiaratchi C, James A, Collins M (1987) Island wakes and headland eddies: a comparison between remotely sensed data and laboratory experiments. J Geophys Res 92(C1):783-794, DOI 10.1029/jc092ic01p00783

24. Raffel M, Willert C, Wereley S (2007) Particle image velocimetry: a practical guide. Springer Verlag

25. Sarpkaya T (1986) Force on a circular cylinder in viscous oscillatory flow at low keulegancarpenter numbers. J Fluid Mech 165:61-71, DOI 10.1017/ s0022112086002999

26. Satijn MP, Cense AW, Verzicco R, Clercx HJH, van Heijst GJF (2001) Threedimensional structure and decay properties of vortices in shallow fluid layers. Phys Fluids 13(7):1932-1945, DOI 10.1063/1.1374936 
27. Scarano F, Poelma C (2009) Three-dimensional vorticity patterns of cylinder wakes. Exp Fluids 47(1):69, DOI 10.1007/s00348-009-0629-2

28. Sciacchitano A, Neal DR, Smith BL, Warner SO, Vlachos PP, Wieneke B, Scarano F (2015) Collaborative framework for piv uncertainty quantification: comparative assessment of methods. Meas Sci Technol 26(7):074004, DOI 10.1088/0957-0233/26/7/074004

29. Signell RP, Geyer WR (1991) Transient eddy formation around headlands. Journal of Geophysical Research: Oceans 96(C2):2561-2575, DOI 10.1029/ 90JC02029

30. Van Dyke M (1982) An album of fluid motion. Parabolic Press Stanford, DOI $10.1115 / 1.3241909$

31. Westerweel J (1997) Fundamentals of digital particle image velocimetry. Meas Sci Technol 8(12):1379, DOI 10.1088/0957-0233/8/12/002

32. Westerweel J, Scarano F (2005) Universal outlier detection for piv data. Exp Fluids 39(6):1096-1100, DOI 10.1007/s00348-005-0016-6

33. Williamson CHK (1985) Sinusoidal flow relative to circular cylinders. J Fluid Mech 155:141-174, DOI 10.1017/s0022112085001756

34. Wolanski E, Hamner W (1988) Topographically controlled fronts in the ocean and their biological influence. Science (New York, NY) 241(4862):177, DOI 10.1126/science.241.4862.177

35. Wolanski E, Imberger J, Heron ML (1984) Island wakes in shallow coastal waters. J Geophys Res 89(C6):10553-10569, DOI 10.1029/jc089ic06p10553

36. Wolanski E, Asaeda T, Tanaka A, Deleersnijder E (1996) Three-dimensional island wakes in the field, laboratory experiments and numerical models. Cont Shelf Res 16(11):1437 - 1452, DOI 10.1016/0278-4343(95)00087-9

37. Wolanski E, Brinkman R, Spagnol S, McAllister F, Steinberg C, Skirving W, Deleersnijder E, et al (2003) Merging scales in models of water circulation: perspectives from the great barrier reef. Advances in Coastal Modeling Elsevier pp 411-429 